selection and testing policies and hence minimising the risk of transfusion transmitted infections. The surveillance programme has evolved to include information on new tests, horizon scanning for emerging infections and microbiological screening of antenatal samples.

Methods Applications of surveillance data for the 15-year period were reviewed. The role in informing transfusion and public health policies, epidemiology and natural history of infections are described.

Results Annual estimates of and trends in incidence and prevalence of infection among donors, donor behaviours associated with infections, non-compliance with selection criteria, and estimates of the risk of transfusion-transmitted infection were determined. These data were used to evaluate and inform policy on microbiological testing and donor selection such as the introduction of nucleic acid testing and the associated risks of changes to deferral criteria. The surveillance programme has expanded into other areas: cohort studies of HCV and HTLV infected patients, look-back studies of transfusion recipients, collaborative work on genotyping and sero-surveillance, and assessments of donor health such as heart disease. The unit also contributes to national surveillance programmes through the notification of infections and informs antenatal screening policy with work on rubella.

Conclusion Blood donors are a sentinel population and surveillance provides a regular and valuable source of epidemiological information about a low-risk population providing opportunities for additional work into the nature of infection. Surveillance programmes within blood services are essential to evaluate and optimise blood safety.

\section{6-4.5 REGULATION WORKS: CONTROLLING NEW ZEALAND'S CAMPYLOBACTERIOSIS EPIDEMIC CAUSED BY CONTAMINATED CHICKEN MEAT}

doi:10.1136/jech.2011.142976b.82

${ }^{1} \mathrm{M}$ Baker, ${ }^{*}{ }^{1} \mathrm{~A}$ Sears, ${ }^{1} \mathrm{~N}$ Wilson, ${ }^{2} \mathrm{~N}$ French. ${ }^{1}$ University of Otago, Wellington, New Zealand; ${ }^{2}$ Institute of Veterinary, Animal and Biomedical Sciences, Massey University, New Zealand

Background The New Zealand epidemic of campylobacteriosis increased steadily from 1989 onwards, peaking in 2006 with a national rate of over 380 notified cases per 100000 population. At the peak there were an estimated 120000 cases a year in the community, and 800 hospitalisations. This rate was markedly higher than that reported by other developed countries. Interventions were introduced to lower contamination levels in fresh chicken meat, notably mandatory monitoring and reporting of Campylobacter in broiler flocks and carcass rinsates, and mandatory Campylobacter carcass performance targets.

Methods National notification and hospitalisation data for the period 1997 to 2008 were analysed to describe disease incidence and distribution. Source attribution techniques based on bacterial typing of Campylobacter isolates from human cases and environmental sources were also used to examine the decline.

Results Directly following implementation of the regulatory measures, the 2008 campylobacteriosis notification rate declined by $54 \%$ and the hospitalisation rate by $56 \%$ (compared to the average annual rates for 2002-2006). Source attribution studies suggested an approximate $70 \%$ decline in human disease with chicken meat as the source.

Conclusions These marked reductions in disease incidence directly followed the introduction of regulatory interventions to reduce Campylobacter contamination of chicken meat. Measures aimed at lowering contamination of raw food appeared far more effective than educational approaches aimed at improving food handling by consumers. Changes to established food production and processing methods may initially be resisted by the food industry, highlighting the need for science-based public health advocacy and regulation. High quality public health surveillance of disease and hazards can also help drive improvements in food safety.

\section{6-4.6 IMPACT OF SCOTTISH SMOKE-FREE LEGISLATION ON USE OF NICOTINE REPLACEMENT THERAPY AND ADULT SMOKING PREVALENCE}

doi:10.1136/jech.2011.142976b.83

${ }^{1} \mathrm{D}$ Mackay, ${ }^{2,3} \mathrm{~S}$ Haw, 'J Pell. 'Public Health Section, University of Glasgow, Glasgow, UK; ${ }^{2}$ Scottish Collaboration for Public Health Research \& Policy, Edinburgh, UK; ${ }^{3}$ NHS Health Scotland, Edinburgh, UK

Introduction In Scotland, legislation was implemented in March 2006 prohibiting smoking in all wholly or partially enclosed public spaces. We investigated the impact on NHS prescriptions for nicotine replacement therapy (NRT) and smoking prevalence.

Methods We analysed monthly data on the gross ingredient cost of all NRT prescribed in Scotland 2003-2009. The Scottish Household Survey provided quarterly data on self-reported smoking status between January 1999 and March 2010. We developed time series models for both datasets using Box-Jenkins autoregressive integrated moving averages.

Results NRT prescription costs were significantly higher than expected over the 3 months prior to implementation of the legislation. Prescription costs peaked at $£ 1.1$ million in March 2006; $£ 231753$ (95\% CI $£ 200800$ to $£ 262707, p<0.001$ ) higher than the monthly norm. Following implementation of the legislation, costs fell exponentially by around $22 \%$ per month (95\% CI $13 \%$ to $32 \%$, $\mathrm{p}<0.001)$. Twelve months following implementation, the costs were not significantly different to monthly norms. Smoking prevalence fell by $6.0 \%$ overall, from $31.3 \%$ in January 1999 to $25.3 \%$ in March 2010. In the quarter prior to implementation of the legislation, smoking prevalence fell by $2.4 \%$ (95\% CI $0.4 \%$ to $4.4 \%$, $\mathrm{p}=0.019)$ more than expected from the underlying trend.

Conclusions Prescriptions for NRT increased in the 3 months prior to Scotland's smoke-free legislation, resulting in a fall in smoking prevalence but neither were sustained. We argue that the early benefits may have been sustained for longer, if the high profile media awareness and education campaigns that preceded the legislation had continued post-legislation.

\subsection{METHODOLOGY AND EPIDEMIOLOGY}

\section{Chair: Dr. Robert West, UK 06-5.1 MILLION DEATH STUDY MORTALITY CLASSIFICATION (MDS-MC) SYSTEM FOR VERBAL AUTOPSIES}

doi:10.1136/jech.2011.142976b.84

${ }^{1} \mathrm{~W}$ Suraweera, ${ }^{2} \mathrm{~V}$ Malhotra, ${ }^{*} \mathrm{D}$ Bassani, ${ }^{1} \mathrm{~S}$ Morris, ${ }^{3} \mathrm{P}$ Mony, ${ }^{3} \mathrm{R}$ Jotkar, ${ }^{1} \mathrm{P}$ Jha. ${ }^{1}$ Centre for Global Health Research, Li Ka Shing Knowledge Institute, St. Michael's Hospital, Dalla Lana School of Public Health, University of Toronto, Toronto, Ontario, Canada; ${ }^{2}$ Centre for Global Health Research (India) Foundation, New Delhi, India; ${ }^{3}$ St. John's Research Institute, St. John's National Academy of Health Sciences, Bangalore, Karnataka, India

Introduction The Global Burden of Disease (GBD) disease classification system, based on ICD-10, is mostly based on hospitalised patients and medically-certified causes of death in developed countries. However, most deaths in developing countries occur at home, without medical attention. Classification systems specific to verbal autopsies are thus needed. The GBD includes ICD codes that cannot be applied to community deaths which often lack detailed clinical 
information and proportionately distributes ill-defined ICD codes among disease groups without explicit guidelines. We propose a modified classification system-Million Death Study mortality classification system (MDS-MC) - that is practicable for use in verbal autopsy studies worldwide.

Methods We use a Delphi method to categorise ICD codes into disease categories. These categories are then structured into broader disease groups. We compare our classification system against GBD and check for age and sex-wise epidemiological plausibility of major diseases using the MDS study results for 123000 deaths from 2001 to 2003 across India.

Results 31 disease groups are divided into four broad disease group$s$-communicable, non-communicable, injury and ill-defined. The MDS-MC produces built-in quality indicators (such as proportion of ill-defined causes for deaths before old age, range checks, etc) that make it easier to manage the inherent misclassification in verbal autopsy. Major disease profiles are epidemiologically plausible for age and sex. A simple to use web-program enables its use in various settings.

Conclusions The MDS-MC, designed specifically for coding of verbal autopsies, is a widely practicable classification system for use in developing countries.

\section{6-5.2 LINKING INDIVIDUAL RECORDS FROM MULTIPLE LARGE DATABASES; A SHORT HISTORY AND PRACTICAL SOLUTION}

doi:10.1136/jech.2011.142976b.85

M Lugg.* Kaiser Permanente, Pasadena, California, USA

Quality epidemiological studies require linked records to differentiate persons from events.

Medical records in the 1960's were mainly linked manually by visually comparing personal data, such as in the Western Australian Mental Health Services Registry.

In 1973, Australia's Hospital and Allied Services Advisory Council (HASAC) studied numerous ways of creating a uniform personal identifier using personal data / attributes which were readily available to the patient and healthcare provider without recalling a medical record number or finding a membership card. Using portions of the last name, first name, gender, and date of birth; only 17 out of 696000 records could not be automatically linked. A tiebreaker, such as place of birth was recommended.

In 1993, the California Health Information for Policy Project's Interagency Working Party, working with 10 different state and local databases, suggested the use of the HASAC algorithm, but used mother's maiden name instead of place of birth to prevent duplicates requiring manual adjudication. This recommendation however could not be implemented practically because many organisations whose records did not include mother's maiden name found the cost of finding this information prohibitive.

In 2003, an attempt was made to further modify the HASAC criteria using only computerised available data to link just under 2 million records for the Kaiser Permanente Southern California Immunisation registry (KITS). As series of 26 computerised algorithms was developed which resulted in a $99.8 \%$ matching rate.

This study resulted in a cost-effected way of routinely linking records from large data bases with minimal manual input.

\section{6-5.3 VASOVAGAL REACTION FROM BLOOD DONATION AND BIOMARKERS IN JAPANESE}

doi:10.1136/jech.2011.142976b.86

${ }^{1} \mathrm{~T}$ Odajima, ${ }^{*}{ }^{1} \mathrm{H}$ Sugimori, ${ }^{2} \mathrm{M}$ Takanashi, ${ }^{2} \mathrm{~S}$ Aota, ${ }^{2} \mathrm{M}$ Sudoh, ${ }^{2} \mathrm{Y}$ Yamaga, ${ }^{2} \mathrm{Y}$ Ono, ${ }^{2} \mathrm{~K}$ Matsuzaki, ${ }^{2,3} \mathrm{M}$ Satake, ${ }^{4} \mathrm{~K}$ Yoshinaga, ${ }^{4} \mathrm{~T}$ Motoji, ${ }^{2} \mathrm{~K}$ Nakajima. ${ }^{1}$ Department of Preventive Medicine, Daito Bunka University Graduate School of Sports and Health
Science, Saitama, Japan; ${ }^{2}$ Japanese Red Cross Tokyo Blood Centre, Tokyo, Japan: ${ }^{3}$ Japanese Red Cross Central Blood Institute, Tokyo, Japan; ${ }^{4}$ Department of Hematology, Tokyo Women's Medical University, Tokyo, Japan

Introduction The Japanese Red Cross Tokyo Blood Center collects approximately 600000 blood donations every year. Since we experienced a fatal injury case related to vasovagal reaction (VVR) syncope in 2005, we conducted a cross-sectional study to elucidate factors contributing to VVR, the most frequent side effect, following whole blood and apheresis donations.

Methods Complications recorded at the collection sites after voluntary donations by Japanese Red Cross Tokyo Blood Center, in the 2006 and 2007 fiscal years, were analysed by univariate and multivariate logistic regression model. Of 1119716 blood donations over a full 2 years, complications were recorded for 13320 donations $(1.189 \%)$, among which VVR was the primary or secondary complication in $67 \%$. Eligible 4303 VVR cases having sufficient information, and 40256 control donors were prepared. Age, body mass index, predonation blood pressure, pulse, and biomarkers (eg, total protein, albumin, and haemoglobin) were compared between VVR group and control group.

Results VVR group was significantly younger, lower body mass index, lower blood pressure, higher biomarkers' values (eg, total protein, albumin, and haemoglobin) than control group. $(p<0.001)$ Furthermore, biomarkers' values and VVR incidences showed a dosedependent manner. (Trend test $\mathrm{p}<0.01$ ).

Conclusion Obviously, to prevent serious consequences, donors should be informed about importance of rest afterwards, and posture to take when symptoms occur. From our analysis, extra care should be considered including high biomarkers' concentrations (eg, total protein, albumin, and haemoglobin), which might reflect donor's dehydration state.

\section{6-5.4 VENTILATOR-ASSOCIATED PNEUMONIA IN PATIENTS WITH SEVERE BURN INJURY: THE PREDICTIVE VALUE OF ROUTINE SURVEILLANCE CULTURES TO PREDICT MULTIDRUG RESISTANCE}

doi:10.1136/jech.2011.142976b.87

${ }^{1,2} \mathrm{~N}$ Brusselaers, ${ }^{*}{ }^{1} \mathrm{D}$ Logie, ${ }^{1,2} \mathrm{D}$ Vogelaers, ${ }^{1,3} \mathrm{~S}$ Monstrey, ${ }^{1,2} \mathrm{~S}$ Blot. ${ }^{1}$ Ghent University, Ghent, Belgium; ${ }^{2}$ Ghent University Hospital, General Internal Medicine, Ghent, Belgium; ${ }^{3}$ Ghent University Hospital, Burn Unit, Ghent, Belgium

Introduction Burn patients with inhalation injury requiring mechanical ventilation $(\mathrm{MV})$ are at particular risk for ventilatorassociated pneumonia (VAP), which is associated with increased morbidity and mortality. Routine endotracheal surveillance cultures (SC) may provide information about the causative pathogen in subsequent VAP, facilitating early appropriate antibiotic therapy.

Objectives To assess the value of routine endotracheal SC to predict multidrug resistant (MDR) aetiology of VAP in burn patients with inhalation injury.

Methods Historical cohort ( $\mathrm{N}=46)$ study including all burn patients with inhalation injury who developed VAP during admission to the burn unit at Ghent University Hospital (2002-2009).

Results Overall, 70 episodes of VAP occurred. Median age and total burned surface area were 43.5 y (IOR 38.0 to 54.3 ) and 32.5\% (18.0 to 45.8$)$ respectively. The median Belgian Outcome in Burn Injury score was 5 (4-6), reflecting a predicted mortality of $30 \%$ (20-50\%). ${ }^{1}$ Median duration of MV prior to onset VAP was $7 \mathrm{~d}$ (4-9d). The incidence of VAP was 55 episodes/1000 MV days and 112 episodes/1000 MV days "at risk." In 23 episodes (32.9\%) at least one MDR causative pathogen was involved (24 MDR pathogens), mostly Pseudomonas aeruginosa (10/23) and Enterobacter spp. (7/23). The sensitivity and specificity of SC to predict MDR pathogens was 